\title{
New recurrent chromosome change in pediatric therapy-related myelodysplastic syndrome: unbalanced translocation $1 / 6$ with cryptic duplication of short arm of chromosome 6
}

\author{
Elisa Tassano ${ }^{1}$, Elisa Tavella ${ }^{1}$, Roberto Valli ${ }^{2}$, Concetta Micalizzi ${ }^{3}$, Cristina Cuoco ${ }^{1}$, Emanuela Maserati ${ }^{2}$, \\ Francesco Pasquali ${ }^{2} \&$ Cristina Morerio ${ }^{1}$ \\ ${ }^{1}$ Cancer Cytogenetic Laboratory and ${ }^{3}$ Department of Pediatric Hematology Oncology, Giannina Gaslini Institute, \\ Genova, Italy and ${ }^{2}$ Department of Experimental and Clinical Biomedical Sciences, University of Insubria, \\ Varese, Italy
}

\begin{abstract}
The incidence of therapy-related myelodysplastic syndrome (t-MDS) in pediatric patients is increasing in parallel with the more successful management of the primary tumor, but scant information is available on clinical and cytogeneticcharacteristics. We report here two children affected by t-MDS after chemo/ radiotherapy for a primary solid tumor, both with an unbalanced translocation $1 / 6$ in their bone marrow. Characterization by array comparative genomic hybridization of the imbalances showed an almost identical pattern: almost complete trisomy of the long arm of chromosome 1, and a terminal deletion and interstitial duplication of the short arm of chromosome 6. The gain of chromosome 6 short arm encompasses regions already highlighted as possibly relevant for t-MDS in adults, and we suggest that the unbalanced translocation reported here be considered a new recurrent, non-random chromosomal abnormality in pediatric patients with t-MDS.
\end{abstract}

Keywords: t-MDS, pediatric age, unbalanced translocation, array-CGH

\section{Introduction}

Therapy-related myelodysplastic syndrome (t-MDS) is a serious complication of cancer treatment. The incidence of pediatric t-MDS is between 7 and $18 \%$ of all childhood MDS, and this incidence has increased in recent years as a result of the increasing number of patients who survive their primary cancer [1]. The outcome of t-MDS is poor; hematopoietic stemcell transplantation (HSCT) has improved the survival rate, but t-MDS is still associated with a significantly increased relapse risk compared to de novo MDS (33.3\% vs. 70\% 5-year relapse-free survival) [2]. MDS is uncommon in children and t-MDS represents only a fraction of patients with MDS, and thus limited and heterogeneous information on the clinical and cytogenetic characteristics is available. Most pediatric patients with t-MDS show an abnormal karyotype in the bone marrow (BM), and loss of chromosome 7 or deletion of chromosome 7 long arm are the most common abnormalities [3]. Unbalanced rearrangements involving the long arm of chromosome 1, with $1 \mathrm{q}$ trisomy, as well as structural changes of the short arm of chromosome 6 , have been reported as recurrent in MDS, in particular in t-MDS [4,5], whereas an unbalanced translocation between chromosomes 1 and 6 with the presence of a derivative chromosome $\operatorname{der}(6) t(1 ; 6)$ (q21 23;p21.3) has been specifically associated with other myeloid disorders, in particular primary myelofibrosis [6]. In t-MDS and therapy-related acute myeloid leukemia ( $\mathrm{t}$-AML), a genomic gain of $5-6 \mathrm{Mb}$ at $6 \mathrm{p} 21$ has been suggested as a new cryptic molecular rearrangement [7].

We report here two pediatric cases of t-MDS associated with the unbalanced translocation $\operatorname{der}(6) \mathrm{t}(1 ; 6)(\mathrm{q} 21 ; \mathrm{p} 22)$, in whom also a cryptic duplication at $6 \mathrm{p} 11.2$-p22.2 was found.

\section{Patients and methods}

\section{Patient 1}

The first patient is male, diagnosed with neuroblastoma stage IVS with $M Y C N$ amplification at 1 month of age in June 2005. Chemotherapy included four cycles of carboplatin/VP16, cyclophosphamide for hematopoietic stem cell mobilization, and busulfan/melphalan prior to autologous HSCT, performed when the child was 5 months old. A few months after the end of chemotherapy, when he was 1 year old, he developed an anaplastic large-cell lymphoma (ALCL) lympho-histiocytic type $(N P M / A L K$ chimeric transcript positive on reverse transcription-polymerase chain reaction [RT-PCR]) and was treated according to the ALCL99 protocol,

Correspondence: Cristina Morerio, Laboratorio di Citogenetica Ematoncologica, IRCCS Giannina Gaslini, Via Gerolamo Gaslini 5, 16147 Genova, Italy. Tel: +39-010-5636656. Fax: +39-010-5636714. E-mail: cristinamorerio@ospedale-gaslini.ge.it 
i.e. with four cycles of ifosfamide, methotrexate, dexamethasone, VP16 and cytarabine at modulated doses due to his previous chemotherapy treatment, plus 4 months of maintenance with weekly vinblastine. One month after therapy discontinuation (December 2006) he developed cutaneous relapse of the lymphoma with molecular positive BM chimeric transcript, and therefore he was treated with cis-retinoic acid for 14 months. All treatments were completed when the patient was 2 years and 10 months old. When aged 3 years (May 2008), 1 month after cis-retinoic acid discontinuation, he presented moderate thrombocytopenia with increased mean platelet volume (MPV) (mean platelet count $80 \times 10^{9}$ cells/L, MPV 9.9 fL). By the age of 5 years (July 2010), he had monocytosis with the presence of immature myeloid cells in peripheral blood (PB) (white blood cells [WBC] $11 \times 10^{9} / \mathrm{L}$, hemoglobin [Hb] $11.9 \mathrm{~g} / \mathrm{dL}$, platelets $76 \times 10^{9} / \mathrm{L}$ ) and increased fetal hemoglobin (HbF; $6 \%$ ). The differential blood count showed $70 \%$ neutrophils, 16\% lymphocytes, $13 \%$ monocytes and $1 \%$ myelocytes. BM aspirate showed myeloid hyperplasia with dysmyelopoiesis, an increase in monocytes, and megakaryocytes with dysmorphic features (Figure 1). Tests for diagnosis of juvenile myelomonocytic leukemia were negative. NRAS, KRAS and PTPN11 oncogene mutations were not found, and there was no spontaneous proliferation of BM and PB mononuclear cells in vitro. The final clinical and hematological picture was compatible with a diagnosis of therapy-related chronic myelomonocytic leukemia (t-CMML). Currently, at the age of 7 years, the patient has clinical stability with mild splenomegaly without therapy.

\section{Patient 2}

Preliminary data on the second patient have already been reported by Morerio et al. [4]. A 7-month-old boy was diagnosed with medulloblastoma and treated according to the UK Children's Cancer Study Group (UKCCSG) CNS 9204 protocol. After surgical resection, chemotherapy (carboplatin, etoposide, cyclophosphamide, vincristine, thiotepa, methotrexate) and radiotherapy $\left(7.2 \mathrm{~Gy}{ }^{60} \mathrm{Co}\right.$, posterior fossa) were administered for 14 months. In January 2002, at 11 years of age, he was referred to us with a history of cold abscesses that responded only to steroids. We hypothesized a diagnosis of "streaking leukocyte syndrome," or PAPA syndrome (pyogenic sterile arthritis, pyoderma gangrenosum and acne). He had a normal white blood cell count (WBC $5.1 \times 10^{9} / \mathrm{L}$ ), mild anemia (Hb $9.7 \mathrm{~g} / \mathrm{dL}, \mathrm{MCV}$ $80 \mathrm{fL}$ ) and thrombocytopenia (platelet count $132 \times 10^{9} / \mathrm{L}$ ). The differential blood count showed $11 \%$ neutrophils, $85 \%$ lymphocytes and 3\% monocytes. The BM was hyperplastic with trilinear myelodysplastic features, numerous abnormal megakaryocytes, hyperplastic and vacuolated myeloid series with partial deficit of myeloperoxidase, and prominent dyserythropoiesis (Figure 2). MDS was classified as refractory cytopenia with multilineage dysplasia. This diagnosis was
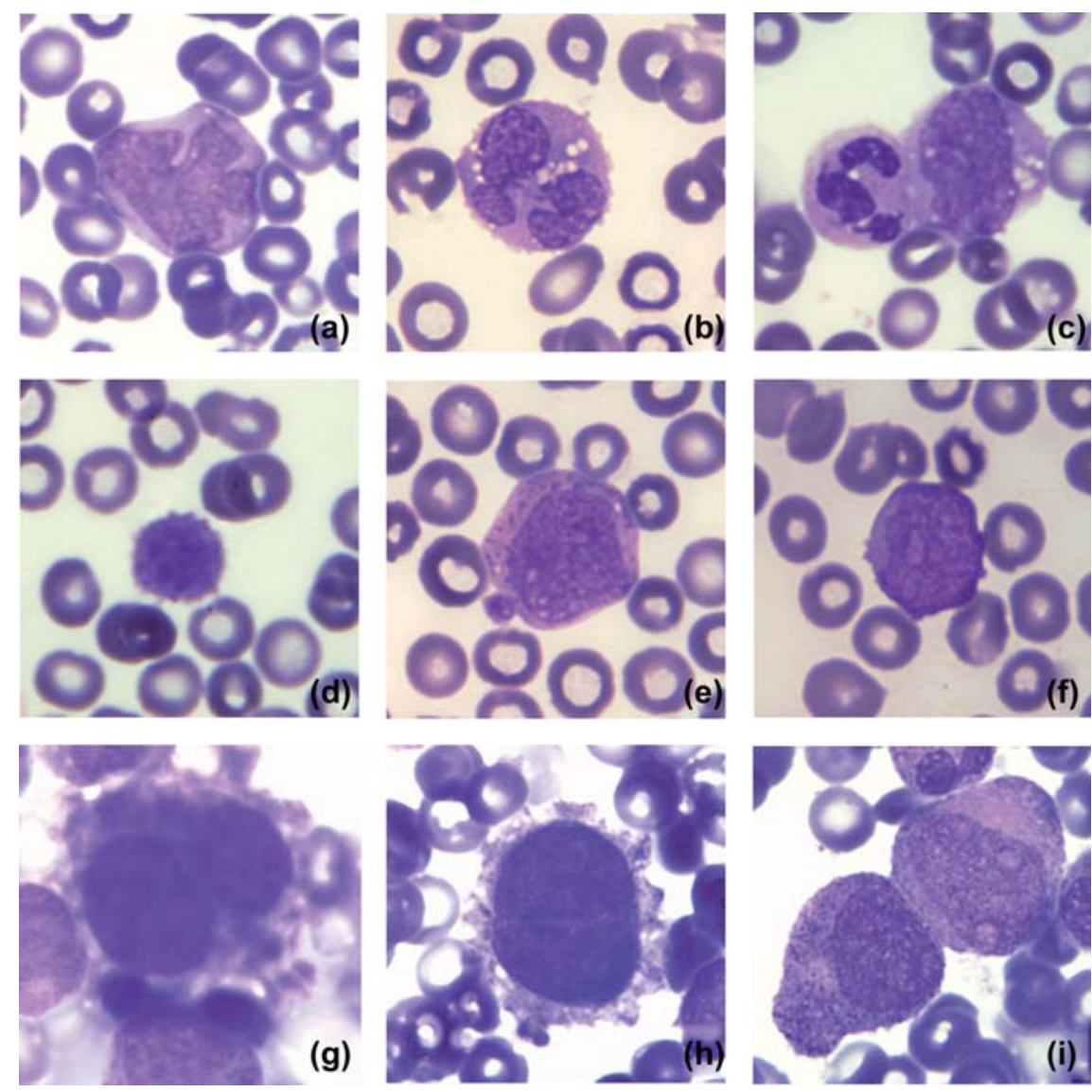

Figure 1. Patient 1 morphologic features (May-Grünwald-Giemsa [MGG], $\times 1000)$ : PB $(a, b, c)$ monocytes with abnormal shape, (d) giant platelet and $(\mathrm{e}, \mathrm{f})$ myeloid precursors; $\mathrm{BM}(\mathrm{g}, \mathrm{h})$ binucleate megakaryoblasts and (i) dysplastic myeloid precursors. 

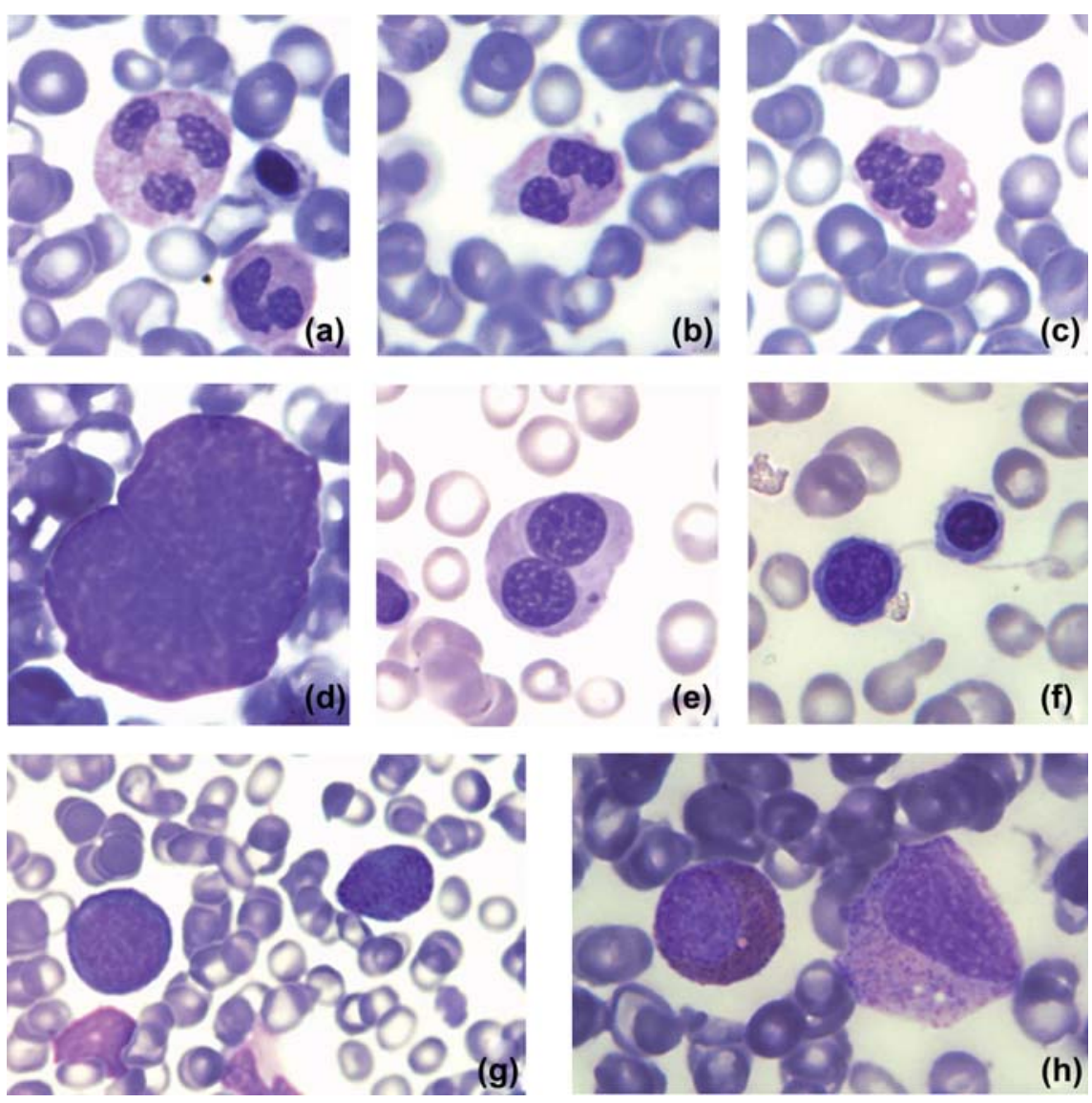

Figure 2. Patient 2 BM morphologic features (MGG, $\times 1000):(a, b, c)$ dysplastic and vacuolated neutrophils, (d) bare megakaryocyte nucleus, $(\mathrm{e}, \mathrm{f}, \mathrm{g})$ evidence of dyserythropoiesis and $(\mathrm{h})$ vacuolated myelocytes.

made 9 years after the end of chemotherapy. Allogeneic BM transplantation from a partially matched unrelated donor failed, and the patient died with disseminated aspergillosis.

\section{Cytogenetics}

Chromosome analyses were performed on unstimulated $\mathrm{BM}$ and $\mathrm{PB}$ cultures from the two patients by QFQ-banding technique. Fluorescence in situ hybridization (FISH) was carried out according to manufacturers' protocols using a library for whole chromosome painting (wcp) and alphoid probes centromere-specific for chromosomes 1 and 6 in both patients.

\section{Array-CGH}

Comparative genomic hybridization on microarray (a-CGH) using a 180K genome-wide system (Agilent Technologies Inc., Santa Clara, CA) with an average resolution of 12-13 kb was performed according to the manufacturer's instructions on DNA from BM sampled in February 2007 in patient 1, when he had normal hematological values and was on cisretinoic acid treatment, and on DNA from BM of patient 2 sampled in September 2002, 9 months after the development of PAPA syndrome.

DNA was extracted using a Qiagen Blood and Tissue Kit (Qiagen GmbH, Hilden, Germany), and competitor DNA was purchased from Promega (Promega Corporation, Madison, WI). Slides were scanned using an Agilent microarray scanner G2565CA, and microarray images were analyzed using Agilent Feature Extraction 10.7.3.1 and Genomic Workbench 5.0.14 software. All a-CGH results refer to the genomic map Hg19.

\section{Results}

\section{Patient 1}

Chromosome analysis carried out on BM at ALCL onset showed a normal karyotype in 16 mitoses analyzed. At the time of cutaneous relapse in December 2006, 19 out of 35 metaphases examined showed an unbalanced translocation between chromosomes 1 and 6, confirmed by wcp, resulting in partial trisomy 1 and partial monosomy 6, and the karyotype was interpreted as follows: $46, \mathrm{XY}, \operatorname{der}(6) \mathrm{t}(1 ; 6)$ (q21;p22)/46,XY (Figure 3). This abnormal clone was found again at six different chromosome analyses performed in 2007-2008, always in the majority of mitoses analyzed. In

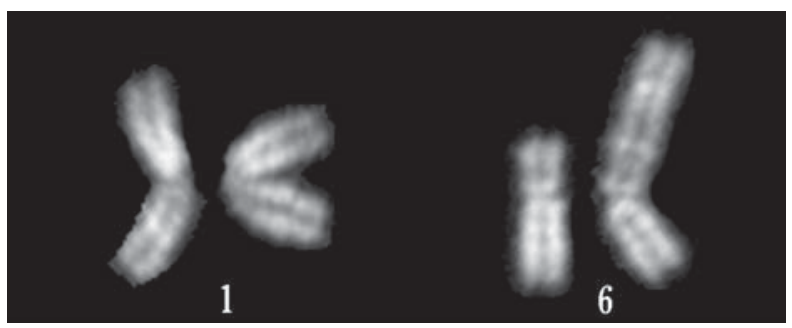

Figure 3. Partial Q-banded karyotype. 
the same period, the $N P M / A L K$ rearrangement gradually disappeared at molecular analysis. Then, in December 2008 and September 2010, all mitoses analyzed on NPM/ALKnegative BM showed the unbalanced translocation (27 and 20 mitoses, respectively).

The imbalances of the abnormal clone were characterized by a-CGH, which showed a gain of material of the long arm of chromosome 1, from band q21.1 to qter (104.2 Mb, 145076 147-249 $212609 \mathrm{bp}$ ), and a loss of chromosome 6 short arm from pter to band p22.2 (26.6 Mb, $170426-26663345 \mathrm{bp}$ ), but also duplication of a segment of chromosome 6 short arm from band p22.2 to band p11.2 (30.2 Mb, $26987363-57$ $190787 \mathrm{bp}$ ) [Figure 4(a)]. The proportion of cells with the three imbalances was evaluated by a previously established formula [8], and the results showed $52-53 \%$ abnormal cells, a percentage compatible with the interpretation that these cells shared the three imbalances.

A homozygous deletion of $104.5 \mathrm{~kb}$ at band q26.1 of chromosome 3 long arm (162 514 534-162 $619082 \mathrm{bp}$ ) was also detected in $100 \%$ of cells, and since it is included in the Database of Genomic Variants (DGV; http://projects.tcag. ca/variation/), updated March 2010, it was interpreted as a constitutional benign copy number variation (CNV).

\section{Patient 2}

The karyotype of BM and PB cells at the onset of t-MDS was 46 , XY, der(6)t(1;6)(q21;p21 22) in all 33 metaphases analyzed. This result was confirmed by FISH with wcp and centromeric probes for chromosomes 1 and 6. Definition by a-CGH of the imbalances showed: gain of material of the long arm of chromosome 1 from band q21.1 to qter (103.95 Mb, 145264 630-249 $212609 \mathrm{bp);} \mathrm{loss} \mathrm{of} \mathrm{chromosome} 6$ short arm from pter to band p22.2 (26.6 Mb, $170426-26663345 \mathrm{bp}$ ); and gain of a segment of chromosome 6 short arm from band p22.2 to band p11.2 (27.3 Mb, 26971 058-54 310034 bp) [Figure 4(b)]. The proportion of cells with the three imbalances, evaluated as above, indicated $67-73 \%$ of abnormal cells, a percentage compatible with the interpretation that these cells shared the three imbalances.

Another two variations were detected, but present in $100 \%$ of cells, and interpreted as a constitutional benign CNV: a heterozygous deletion of $74.9 \mathrm{~kb}$ in band q28.2 of chromosome 4 long arm (129 342 168-129 417126 bp), not included in the DGV mentioned above, and a heterozygous deletion of $56.1 \mathrm{~kb}$ in band q22.3 of chromosome 10 long arm (79 743739 $79799851 \mathrm{bp}$ ), included in the DGV as an inversion variation.

\section{Discussion}

Complete or partial trisomy of the long arm of chromosome 1 is a recurrent anomaly of pediatric MDS both de novo and therapy-related, in most cases secondary to an unbalanced translocation where the more frequent partner is chromosome 7 [4]. A wide literature survey on cytogenetic abnormalities involving chromosome 1 in myeloid neoplasms has highlighted the chromosomal regions 1q21 32, 1p11 13 and 6 p21.3 as potential loci for harboring genes involved in the development and progression of myeloid malignancies [9].

Rearrangements of the short arm of chromosome 6, both balanced and unbalanced, with breakpoints at bands p21 23, have been described in different types of hematological malignancies induced by mutagens in adults. In the majority of cases, this anomaly is present in the context of a complex karyotype, while only in rare cases it has been observed as the sole abnormality $[5,10,11]$. Although the presence of other chromosomal changes might also suggest that the $6 p$ rearrangement plays a role as a secondary event in development of the neoplastic process, La Starza et al. demonstrated recurrence of the gain of a 5-6 Mb genomic segment at 6p21 in eight cases of t-MDS/t-AML, including a patient with Fanconi anemia. Notably, five out of eight patients with $6 \mathrm{p}$ gain had an unbalanced translocation resulting also in $6 \mathrm{p}$ terminal deletion. The authors suggest that this kind of $6 p$ rearrangement is a major pathogenetic event arising from acquired and/or congenital genomic instability [7].

Terminal deletion and interstitial duplication of the short arm of chromosome 6 have been reported in an a-CGH study in two adult patients with AML at relapse [12], and an unbalanced karyotype 46,XX,der(6)t(1;6)(q12;p22.2), probably with a partial duplication of $6 \mathrm{p}$, was detected by chromosome analysis in two girls with t-MDS following treatment for a primary Ewing/primitive neuroectodermal tumor (PNET) $[13,14]$.

The two patients reported here were affected by t-MDS arising after chemo/radiotherapy for a primary solid tumor. Both cases showed an unbalanced translocation 1/6 resulting
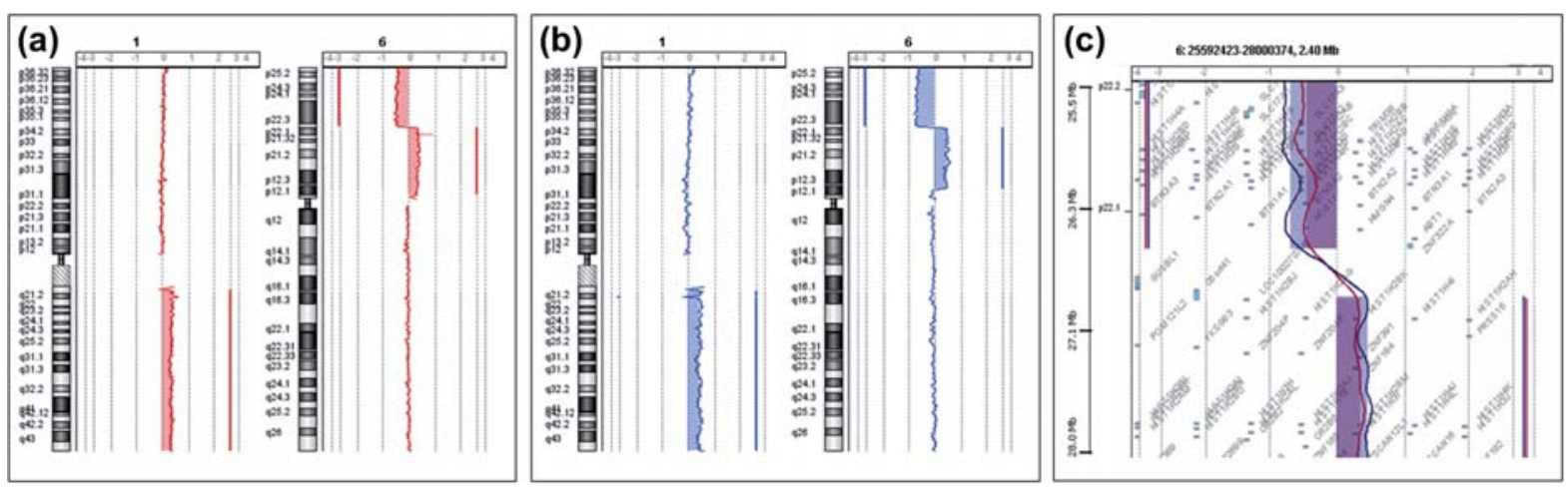

Figure 4. a-CGH profiles of chromosomes 1 and 6 in (a) patient 1 and (b) patient 2, showing gain of material of chromosome 1 long arm, and lost and duplicated regions of chromosome 6 short arm; (c) magnification of the superimposed profiles of both patients (purple, patient 1 ; light gray, patient 2) at the loss/duplication boundary region of chromosome 6 short arm. 
in almost complete trisomy of the long arm of chromosome 1 , monosomy of a segment of $26.6 \mathrm{Mb}$ of the short arm of chromosome 6, and an unexpected partial trisomy of 27-30 $\mathrm{Mb}$ of the short arm of chromosome 6 .

Patient 1 deserves a specific comment because the unbalanced translocation was detected in BM at relapse of the ALCL, 4 years and 6 months before the diagnosis of $t$-CMML. However, at that time no morphologic evidence of lymphoma cells was present in his BM, notwithstanding the fact that the NPM/ALK rearrangement which characterized the ALCL was still detectable. In the following years, this rearrangement disappeared while the $t(1 ; 6)$ clone persisted: in the analyses performed in 2008 and 2010, BM and PB were $N P M / A L K$-negative, and all BM mitoses bore the translocation. Evidence is thus sufficient to conclude that the clone with the translocation had no association with the ALCL, and that it eventually led to the t-MDS.

Patient 2 developed MDS and the unbalanced translocation 1/6, 9 years after stopping chemotherapy. Some studies in the literature have reported cases with chromosomal abnormalities including partial trisomy $1 \mathrm{q}$ and $6 \mathrm{p} 12 \sim 22$ rearrangements identified during continuous complete clinical and morphologic remission of AML, and preceding by months the relapse or appearance of MDS features $[12,15,16]$. Our patients, as well as the two girls with t-MDS mentioned above $[13,14]$, with whom they share the unbalanced karyotype 46,XX,der(6)t $(1 ; 6)(q 12 ; p 22.2)$, were treated with polychemotherapy including etoposide. The risk of secondary leukemia or MDS after a solid tumor in childhood has been shown to increase significantly with the cumulative dose of etoposide [17]; however, the rarity of this chromosomal rearrangement does not allow any correlation with the specific cytotoxic drug.

The occurrence of chromosomal rearrangements involving 1q21 32 and 6 p12 22 observed in secondary malignancies might be the result of architectural features within these regions creating instability, as in the case of various genomic disorders. In several cases, segmental duplications, AT-rich palindromes and pericentromeric repeats were shown to be located at tumor-specific rearrangement breakpoints, as in the genesis of $\mathrm{i}(17 \mathrm{q})$ and the Philadelphia chromosome by translocation $\mathrm{t}(9 ; 22)(\mathrm{q} 34 ; \mathrm{q} 11)[18,19]$.

What is really peculiar in the two cases reported here is the fact that the imbalances due to the translocation were strictly similar, as shown by a-CGH. In particular, apart from trisomy of chromosome 1 long arm, partial monosomy 6 was really identical, whereas the partial trisomy was closely similar; only the gained regions differed slightly from each other, but only toward the centromere, being in fact identical at the point at which they began distally [Figure 4(c)]. The duplicated segment of chromosome 6 short arm encompassed both regions suggested as relevant and identified by FISH and by bacterial artificial chromosome (BAC) a-CGH in previous studies concerning adult patients $[7,12]$. Therefore, at the level of the oligomer-based a-CGH system used, we have described and characterized a new recurrent chromosome change in pediatric t-MDS: an unbalanced translocation 1/6 leading to $\operatorname{der}(6) \mathrm{t}(1 ; 6)(\mathrm{q} 21 ; \mathrm{p} 22.2) \operatorname{dup}(6)(\mathrm{p} 12.1 \mathrm{p} 22.2)$, with a cryptic duplication of the short arm of chromosome 6 .
We also suggest the possibility of detecting this abnormal clone months/years before the appearance of morphological MDS features.

Potential conflict of interest: Disclosure forms provided by the authors are available with the full text of this article at www.informahealthcare.com/lal.

\section{References}

[1] Niemeyer CM, Baumann I. Myelodysplastic syndrome in children and adolescents. Semin Hematol 2008;45:60-70.

[2] Andolina JR, Kletzel M, Tse WT, et al. Allogeneic hematopoetic stem cell transplantation in pediatric myelodysplastic syndromes: improved outcomes for de novo disease. Pediatr Transplant 2011;15:334-343.

[3] Aguilera DG, Vaklavas C, Tsimberidou AM, et al. Pediatric therapyrelated myelodysplastic syndrome/acute myeloid leukemia: the MD Anderson Cancer Center experience. J Pediatr Hematol Oncol 2009;31:803-811.

[4] Morerio C, Rapella A, Tassano E, et al. Gain of 1q in pediatric myelodysplastic syndromes. Leuk Res 2006;30:1437-1441.

[5] Mecucci C, Michaux JL, Louwagie A, et al. The short arm of chromosome 6 is nonrandomly rearranged in secondary myelodysplastic syndromes. Cancer Genet Cytogenet 1988;31:147-155.

[6] Dingli D, Grand FH, Mahaffey V, et al. Der(6)t(1;6)(q21-23;p21.3): a specific cytogenetic abnormality in myelofibrosis with myeloid metaplasia. Br J Haematol 2005;130:229-232.

[7] La Starza R, Aventin A, Matteucci C, et al. Genomic gain at 6p21: a new cryptic molecular rearrangement in secondary myelodysplastic syndrome and acute myeloid leukemia. Leukemia 2006;20:958-964.

[8] Valli R, Maserati E, Marletta C, et al. Evaluating chromosomal mosaicism by array comparative genomic hybridization in haematological malignancies: the proposal of a formula. Cancer Genet 2011;204:216-218.

[9] Caramazza D, Hussein K, Siragusa S, et al. Chromosome 1 abnormalities in myeloid malignancies: a literature survey and karyotype-phenotype associations. Eur J Haematol 2010;84:191-200.

[10] Huret JL, Schoenwald M, Brizard A, et al. Chromosome $6 \mathrm{p}$ rearrangements appear to be secondary changes in various haematological malignancies. Leuk Res 1989;13:819-824.

[11] Mancini M, Mecucci C, Cedrone M, et al. Unbalanced 6p translocation as primary karyotypic anomaly in secondary acute nonlymphocytic leukemia. Cancer Genet Cytogenet 1992;60:93-95.

[12] Tchinda J, Dijkhuizen T, Vlies Pv P, et al. Translocations involving 6 p22 in acute myeloid leukaemia at relapse: breakpoint characterization using microarray-based comparative genomic hybridization. $\mathrm{Br} \mathrm{J}$ Haematol 2004;126:495-500.

[13] Mathew S, Head D, Rodriguez-Galindo C, et al. Trisomy of the long arm of chromosome 1 resulting in a dicentric derivative $(6) \mathrm{t}(1 ; 6)$ chromosome in a child with myelodysplastic syndrome following treatment for a primitive neuroectodermal tumor. Leuk Lymphoma 2000;37:213-218.

[14] Navid F, Billups C, Liu T, et al. Second cancers in patients with the Ewing sarcoma family of tumours. Eur J Cancer 2008;44:983-991.

[15] Raynaud SD, Brunet B, Chischportich M, et al. Recurrent cytogenetic abnormalities observed in complete remission of acute myeloid leukemia do not necessarily mark preleukemic cells. Leukemia 1994;8:245-249.

[16] Michalová K, Lemez $\mathrm{P}$, Bartsch $\mathrm{O}$, et al. Derivative $(6) \mathrm{t}(1 ; 6)$ (q22;p21) revealed in bone marrow cells by FISH 9 months before diagnosis of acute T-lymphoblastic leukemia. Cancer Genet Cytogenet 1996;86:131-135.

[17] Le Deley MC, Leblanc T, Shamsaldin A, et al. Risk of secondary leukemia after a solid tumor in childhood according to the dose of epipodophyllotoxins and anthracyclines: a case-control study by the Société Française d'Oncologie Pédiatrique. J Clin Oncol 2003;21: 1074-1081.

[18] Barbouti A, Stankiewicz P, Nusbaum C, et al. The breakpoint region of the most common isochromosome, $\mathrm{i}(17 \mathrm{q})$, in human neoplasia is characterized by a complex genomic architecture with large, palindromic, low-copy repeats. Am J Hum Genet 2004;74:1-10.

[19] Albano F, Anelli L, Zagaria A, et al. Genomic segmental duplications on the basis of the $t(9 ; 22)$ rearrangement in chronic myeloid leukemia. Oncogene 2010;29:2509-2516. 